\title{
Los refugiados Rohingyas: paradigmas del racismo contemporáneo desde una perspectiva biopolítica
}

\author{
Facundo Ariel Nuñez \\ Estudiante del Profesorado y la Licenciatura en Filosofía \\ Universidad Nacional del Nordeste \\ fan91091@gmail.com
}

\section{Resumen}

A mediados del año 2017 se llevó a cabo un éxodo multitudinario: se trataba de la población Rohingya, quienes a fuerza de asesinatos, incendios programados y violaciones, debieron abandonar la tierra de sus antepasados, forzándolos a buscar suerte en países vecinos donde no pueden ser recibidos y/o son rechazados. Este fenómeno nos permite analizar el panorama político moderno, y recurriendo a los desarrollos teóricos de Michel Foucault y Giorgio Agamben - en particular los conceptos de Estado de excepción y nuda vida de este último, así como también los desarrollos sobre racismo llevados a cabo por el primero -, podemos afirmar que este éxodo es un ejemplo paradigmático de la imbricación fundamental entre racismo y biopolítica.

BIOPOLÍTICA, RACISMO, NUDA VIDA, ESTADO DE EXCEPCIÓN, ROHINGYA.

\section{Abstract}

In mid-2017, a massive exodus was carried out: It was the Rohingya's population who, due to assassinations, programmed fires and rapes, had to leave their ancestors' land, making them try their luck in neighbour countries where they cannot be received and/or they are rejected. This phenomenon allows us to analyze the modern politic panorama, and turning to Michel Foucault and Giorgio Agamben's theoretical developments - particularly the 'State of Exception' and 'Bare Life' concepts of the latter, as well as the works about racism carried out by the former -, we can confirm that this exodus is a paradigmatic example of the essential imbrication between racism and biopolitics.

BIOPOLITICS, RACISM, BARE LIFE, STATE OF EXCEPTION, ROHINGYA. 


\section{Introducción}

En el presente artículo desarrollaremos el éxodo del pueblo Rohingya, presentado como un paradigma de la segregación racial contemporánea, desde una perspectiva biopolítica. Para los Rohingya Birmania ha sido la tierra de sus antepasados desde incontables generaciones, pero a mediados del año pasado fueron obligados a desalojar sus hogares - los cuales luego fueron incendiados por las fuerzas militares birmanas - y refugiarse en Bangladesh o Malasia. Es un caso que escapó a los ojos de muchos medios de comunicación, pero se estima que entre un millón y un millón y medio de personas habitaban en Rakhine, Birmania, de los cuales entre ochocientos mil y un millón lograron escapar, y el resto fue asesinado por las fuerzas militares.

Hemos dicho que pretendemos analizar este caso desde una perspectiva biopolítica, para lo cual recurriremos a las obras de Michel Foucault y Giorgio Agamben, intentando lograr una complementación entre los desarrollos de estos autores. Nos interesa sobre todo la noción de racismo en Foucault, pues es la que posibilita el analisis del pueblo Rohingya como un paradigma contemporáneo de la segregación racial. Estas observaciones sobre el racismo serán puestas en relación directa con el concepto de biopolítica, y a este último iremos añadiendo los aportes de Agamben con el fin de lograr una visión más actualizada. De esta forma, al finalizar, tendremos un complejo sistema de conceptos interrelacionados, los cuales se referirán al caso específico que analizamos y harán más fácil su visualización como ejemplo general.

El centro de la cuestión, como se podrá observar en el desarrollo, es la segregación racial. Normalmente tendemos a pensar que el racismo es un tema del pasado, pero el pueblo Rohingya y la actualidad internacional nos demuestran que no, que es un tema que permanece en la sociedad y quizás con más fuerza que nunca si tenemos en cuenta, por ejemplo, el avance de la ultraderecha en Europa y la elección de Donald Trump en Estados Unidos, así como también las migraciones masivas y la globalización en general. En este contexto, un análisis complejo del fenómeno nos parece no solo necesario, sino más bien imprescindible, y para tal fin decidimos utilizar 
la perspectiva biopolítica pues esta posa sus esfuerzos teóricos en el rol que ocupa la vida en esta clase de situaciones.

Teniendo en cuenta todo lo dicho y lo que subyace a esas palabras, podríamos enunciar nuestra hipótesis de la siguiente manera: "el racismo llevado a sus más funestas consecuencias no es un asunto del pasado, no terminó con los campos de concentración. El pueblo Rohingya, tomado como paradigma de la segregación racial, nos permite visualizar cómo las regulaciones de un Estado pueden tender a eliminar a un pueblo entero: exterminarlo, negarlo u obligarlo a que abandone el territorio de sus antepasados para buscar refugio en tierras inciertas".

El desarrollo del trabajo estará organizado en tres partes generales y una de conciliación y reunión de las anteriores, en la primera de estas partes desarrollaremos la situación del pueblo Rohingya así como también un poco de su historia, en la segunda entraremos en los aportes de Michel Foucault y en la tercera pasaremos a Giorgio Agamben. El momento de conciliación se complementa con la conclusión y dan un cierre al tema poniendo cada tema en relación con los demás, logrando de esta forma un red de conceptos que tiene un reflejo en el caso Rohingya, lo que facilitará el visualizarlo como paradigma.

\section{Historia del pueblo Rohingya}

El pueblo Rohingya, actualmente exiliado en Bangladesh, posee una larga historia de luchas y conflictos que, según algunas fuentes, podría remitirse incluso hasta el siglo VII de nuestra era, con la llegada de los primeros pobladores musulmanes a Birmania. Pero junto con la historia oficial del pueblo, también tenemos el racconto llevado a cabo por las autoridades birmanas, el cual brinda un marcado contraste e intenta exhibir al pueblo Rohingya como invasores ilegales de sus tierras, lo que justificaría la persecución a la cual se somete a este pueblo musulmán.

En este sentido, el artículo Myanmar: Who are the Rohingya? Inicia con estas palabras "The Rohingya are often described as 'the world's most persecuted minority'."19 (AA.VV., 2017) Nurul Islam (2006), presidente de la Arakan Rohingya

\footnotetext{
19 “Los Rohingya son descritos a menudo como 'la minoría más perseguida del mundo”"
} 
National Organisation afirma que su pueblo cuenta entre sus ancestros gente árabe, pashtún (afganos), moros y mongoles, los cuales se asentaron en Arakan (este es el nombre que recibe el territorio de Birmania para la comunidad Rohingya, el cual previamente era conocido como Rohang, de allí el nombre del pueblo) alrededor del siglo séptimo.

Ahora bien, durante los más de cien años de dominio británico sobre la zona, en un período que va de 1824 hasta 1948, se pudo apreciar un incremento en la migraciones de obreros provenientes desde la India y Bangladesh. Estas migraciones fueron consideradas clandestinas e ilegales por la población nativa, motivo por el cual, cuando Birmania alcanzó la independencia del yugo británico, negó la nacionalidad a aquellas minorías consideradas invasoras. Lo curioso del caso es que incluyeron entre estas minorías no reconocidas al pueblo Rohingya, afirmando que los mismos provenían de Bangadlesh y que el término "rohingya" como tal es un invento político moderno.

En realidad es necesario hacer una salvedad, de acuerdo al testimonio de Nurul Islam (2006), pues el pueblo Ronhingya fue originalmente reconocido por las autoridades birmanas como una etnia indigna, pese a lo cual se les permitió enviar sus representantes al parlamento. Pero esta situación cambió a partir de 1962 cuando las fuerzas militares avanzaron sobre el pueblo, iniciando una de las más sangrientas y prolongadas persecuciones de la historia contemporánea. La educación les fue negada de forma indirecta debido a la discriminación y a la situación de pobreza en la que se encontraban inmersos, pues a muchos de ellos no se les permitía comerciar, y los que podían hacerlo debían compartir sus ganancias; los impuestos sobre sus tierras eran cada vez más altos y poco a poco fueron expropiados de las mismas. Situación que se agravó considerablemente en 1982 cuando se elaboró una nueva ley de ciudadanía que los excluía completamente como parte de las 140 etnias reconocidas por el gobierno birmano. El artículo Myanmar: Who are the Rohingya? sentencia "As a result 
of the law, their rights to study, work, travel, marry, practice their religion and access health services have been and continue to be restricted." ${ }^{20}$ (AA.VV., 2017)

\section{Situación actual de los exiliados Rohingya}

Hemos adelantado en el capítulo anterior que hoy en día la población Rohingya se encuentra refugiada en Bangladesh, pero esto solo es cierto para un porcentaje, quizás el más afortunado, de la población que ha escapado del estado de Rakhine en Birmania (lugar donde se llevaron a cabo los mayores ataques contra la población Rohingya y donde habitaba el mayor porcentaje de su población), muchos de ellos fueron rechazados por fuerzas policiales al intentar ingresar en Bangladesh, otros corrieron una suerte similar al intentar refugiarse en Malasia, mientras que una cantidad solo estipulable se encuentra en una situación aun peor: De acuerdo al artículo Myanmar: Who are the Rohingya? "More than 500,000 people have fled the violence, with thousands trapped in the no-man's land between the two countries, according to the UN refugee agency" ${ }^{21}$ (AA.VV., 2017) en otros artículo de Al Jazeera se habla de 650.000 exiliados, o de 800.000 .

Más allá de las cifras exactas, lo cierto es que la población Rohingya debió exiliarse de las tierras que han habitado por cerca de catorce siglos, y hoy en día se encuentran dispersos y en diferentes situaciones, ninguna de las cuales es positiva. El campo de refugiados de Bangladesh, en Cox's Bazar, es el que brinda mayor información acerca de la actualidad del pueblo Rohingya, al menos de aquellos que han logrado escapar a la persecución. Motivo por el cual seguiremos estas noticias, recordando que no representa a la totalidad del pueblo exiliado, y que miles de ellos se encuentras efectivamente en situaciones peores (por ejemplo aquellos atrapados entre fronteras, quines no son admitidos en ningún país).

Durante los últimos meses se ha intentado que Bangladesh y Birmania colaboren en un plan de repatriación de los refugiados Rohingyas, pero si recordamos lo antes desarrollado, aun antes de su exilio esta minoría musulmana carecía de patria,

20 "Como resultado de la ley, sus derechos a estudiar, trabajar, viajar, contraer matrimonio, practicar su religión y el acceso a los servicios de salud fue y continúa siendo restringido."

21 "Más de 500.000 personas han escapado de la violencia, con miles atrapados en tierra de nadie entre los dos países, de acuerdo a la agencia de refugiados de la ONU." 
pues el gobierno de corte mayoritariamente budista, al igual que la población, no los reconocían como ciudadanos oficiales. Y sumada a esta ironía se encuentra un segundo impedimento, el cual se encuentra reflejada en los testimonios recogidos por Judah, J. (2017) en el artículo Soaked and hungry Rohingya refugees seek shelter in Bangladesh:

"From the summit of the hill, you can see across the border into Myanmar. A plume of thick white smoke rises and mixes with the monsoon-heavy clouds. "The village is burning," says Jaheed. Testimonies from those who have just arrived say that there is nothing left."22

Los incendios provocados por las fuerzas militares como medida de persecución no son algo nuevo para el pueblo Rohingya, en el fondo son solo otro testimonio de la situación de abandono en la que se encuentra esta población: sin lugar a donde ir, y sin lugar a donde volver.

Sumemos a esta situación los múltiples reportes de las Naciones Unidas que, a través de sus diferentes organismos, está tratando de ayudar a la población refugiada. El analfabetismo y exclusión de los Rohingya de los planes de salud en Birmania tienen un impacto directo en la taza de nacimientos, y el gobierno de Bangladesh teme que el campo de refugiados crezca antes de reducirse. Otra preocupación del gobierno y de la población bengalí, directamente relacionada con la anterior, es la situación de hacinamiento de la población refugiada, lo cual podría provocar una epidemia infecciosa. Como resultado de la creciente ola de refugiados - de acuerdo al artículo Bangladesh to build one of world's largest refugee camps for 800,000 Rohingya (AA.VV., 2017) - las autoridades de Bangladesh pusieron en marcha múltiples proyectos de ampliación de los campos de refugiados, pero como establecen autoridades de las naciones unidas en ese mismo artículo las necesidades de los Rohingya están creciendo a un ritmo mayor que la habilidad de aquellos para dar con ellas.

22 "Desde la cima de la colina, se puede ver al otro lado de la frontera en Myanmar. Una columna de humo espeso y blanco se eleva y se mezcla con las nubes monzónicas. 'La aldea está ardiendo', dice Jaheed. Los testimonios de quienes acaban de llegar dicen que ya no queda nada." 
Lo dicho alcanza para comprender la situación actual de los refugiados en Bangladesh, los múltiples problemas que afrontan y la incapacidad de los organismos internacionales para satisfacer las necesidades más básicas de una población sin tierra y sin derechos, adelantándonos al desarrollo del presente trabajo: quizás los Rohingya sean el ejemplo más claro en nuestros días de nuda vida.

\section{Situación previa al éxodo}

Como hemos visto en el primer capítulo, la situación de los Rohingyas se ha ido agravando desde la independencia de Birmania en 1948, momento en el cual fueron reconocidos como una minoría étnica indigna, pasando por 1962 cuando iniciaron las persecuciones militares, los asesinatos, los incendios, las violaciones y llegando a 1982 cuando la ciudadanía les fue negada y el pueblo Rohingya permaneció dentro de un territorio que no lo reconocía como perteneciente a él, impidiéndoles o limitándoles el acceso a educación, salud, trabajo y otros derechos fundamentales.

Esta situación ya ha sido descrita en el presente trabajo y lo anterior es a modo de resumen, pues dada la situación en la que se encontraba el pueblo desde mediados del siglo XX es interesante el pensar qué pudo haber provocado este gran éxodo a mediados del 2017. La respuesta no agrega nada nuevo, fueron las persecuciones constantes, la incineración intencional de casas y personas, el asesinato programado y las campañas de violación, todos estos hechos llevados a cabo por las fuerzas militares y policiales de Birmania.

Por supuesto, hay una supuesta justificación para estos hechos atroces, retomando el artículo Myanmar: Who are the Rohingya? "After the killings of nine border police in October 2016, the government blamed what it claimed were fighters from an armed Rohingya group and troops started pouring into the villages of Rakhine State $^{\prime 23}$ (AA.VV., 2017) Este fue el punto de quiebre para la población de origen musulmán, justificados por ese supuesto ataque - y la determinación de los Rohingya como extranjeros ilegales - las fuerzas birmanas llevaron a cabo ejecuciones

23 “Después de los asesinatos de nueve policías fronterizos en octubre de 2016, el gobierno culpó a los que alegaban eran combatientes de un grupo Rohingya armado y las tropas comenzaron a penetrar en las aldeas del estado de Rakhine." 
extrajudiciales, campañas de violación e incineración: un verdadero estado de excepción, como veremos más adelante.

Quizás uno de los puntos que más llamó la atención de la prensa ha sido la confesión, por parte de las autoridades militares de Birmania, del asesinato de diez combatientes Rohingyas, el artículo 'Beyond comprehension': Myanmar admits killing Rohingya recoge el siguiente hecho: "In a Facebook post on Wednesday, Min Aung Hlaing, the military's commander in chief, said soldiers and villagers had confessed to killing 10 suspected Rohingya fighters on September 2." ${ }^{24}$ (AA. VV., 2018) Una vez más, la amenaza interna justifica la utilización de la fuerza, y el asesinato y la detención sin un proceso legal transparente se convierten en las herramientas del Estado.

Los testimonios y los reportes abundan respecto a las atrocidades cometidas por el gobierno de Birmania, hasta el punto que, de acuerdo al artículo Myanmar's Crackdown on Rohingya Is Ethnic Cleansing, Tillerson Says (Landler, M., 2017) estos hechos han sido considerados limpieza étnica por las autoridades del gobierno de los Estados Unidos, el cual buscará duras sanciones para la cúpula militar de Birmania por llevar a cabo esta campaña de terror y destrucción sobre una población minoritaria.

A modo de resumen, el gobierno y la población birmana no consideran a los Rohingya como ciudadanos de su territorio, esto es debido a las dos versiones respecto al origen de los mismos (los habitantes de Birmania ven a los Rohingyas como extranjeros llegados a sus tierras durante la colonización británica, mientras que estos se ven como habitantes legítimos del territorio), y también a la diferencia en sus creencias, mientras el gobierno y la población son en su mayoría budista, los Rohingya son musulmanes. Básicamente, la población y el gobierno de Birmania ve en esta minoría un enemigo interno, un extranjero que amenaza su estilo de vida - las poblaciones musulmanas son constantemente vistas como un peligro potencial en diferentes puntos del mundo, en gran medida debido a ciertas interpretaciones en el interior de la comunidad musulmana del concepto de yihad que han llevado al nacimiento de agrupaciones terroristas, además de la constante guerra entre facciones

\footnotetext{
$24 \quad$ "En una publicación de Facebook el miércoles, Min Aung Hlaing, comandante en jefe del ejército, dijo que soldados y aldeanos confesaron haber matado a 10 presuntos combatientes Rohingya el 2 de septiembre."
} 
en el interior del movimiento musulmán - y que por lo tanto debe ser borrado de su territorio. Interpretamos ahora estos hechos a la luz de la biopolítica.

\section{Génesis del racismo}

En los capítulos anteriores hemos desarrollado la historia del pueblo Rohingya, desde los testimonios de su origen hasta su situación actual de exilio y fragmentación. Hemos dicho que es nuestra intención de ahora en más el analizar esos hechos a la luz de los aportes de la biopolítica, pero antes de avanzar por ese camino nos gustaría detenernos para analizar el surgimiento del discurso racista, y de esta forma ver cómo se articula este con el análisis biopolítico. Para tal fin recurriremos a los aportes de Michel Foucault, pero no sin recordar que, pese a que este sea un tema recurrente en algunos pasajes de las obras del filósofo francés, "el hecho de que el racismo no sea el problema explícito que aborda Foucault le da a sus observaciones cierto aire fragmentario" (Gómez Izquierdo, 2014, p. 121).

Iniciemos el análisis con una cita del autor: "Cuando la guerra fue expulsada a los límites del Estado, centralizada a la vez en su práctica y rechazada a su frontera, apareció cierto discurso: un discurso extraño, novedoso." (Foucault, 2000, p. 54) Expliquémosla brevemente: uno de los primeros avances de la modernidad es la creación de los ejércitos, la guerra se convierte en un asunto de Estado y se la expulsa del interior de las ciudades a sus fronteras. Estamos a finales del siglo XVI, en la antesala de las grandes revoluciones europeas, en las cuales este discurso descripto por Foucault jugará un papel fundamental. Se trata del discurso de la guerra de razas, pero no entendida como la lucha de una raza superior por dominar a las inferiores, se trata más bien de un discurso histórico y con un fuerte impacto político.

Tradicionalmente, durante la edad media y gran parte de la modernidad, se mantuvo una imagen piramidal de la organización estatal, esto lo vemos en el Leviathán de Hobbes, por ejemplo, donde el soberano, como cabeza de un gran organismo creado por todos los individuos que delegan en él una cuota de su libertad, dirige el Estado. Frente a esta visión centralizada y piramidal, el discurso de la lucha de razas propondrá una nueva perspectiva, en la cual "una estructura binaria atraviesa la 
sociedad" (Foucault, 2000, p. 56). Como hemos dicho antes, no es un discurso de tipo racista-biológico, sino que más bien define la estructura de la sociedad, la cual se encuentra conformada por al menos dos sectores que poseen lenguajes, costumbres, ideas y hasta linajes distintos, y cada uno de ellos intenta defender o exigir los derechos que le corresponden a su sector, basándose en un estudio minucioso de la historia, la cual a partir de entonces cobra un papel fundamental, se convierte en la herramienta que permite criticar el supuesto derecho absoluto del soberano, el cual se defiende, a su vez, poniendo en juego otra serie de discursos de verdad basados en otra interpretación de la historia.

Hemos dicho anteriormente que la guerra se desplazó a las fronteras de la sociedad, pues bien, ahora vemos que esto no es totalmente cierto.

"Esta idea, la de que la sociedad está recorrida de uno a otro extremo por este enfrentamiento de las razas, se formula en el siglo XVII y será la matriz de todas las formas bajo las cuales, de allí en adelante, se buscarán el rostro y los mecanismos de la guerra social." (Foucault, 2000, p. 64)

Aquel que enuncia, aquel que dice su verdad - pues la verdad ha dejado de ser absoluta para convertirse en una herramienta - se encuentra en una posición determinada dentro del campo, tiene enemigos, tiene aliados y es imposible que tome una postura neutral o universal como pretendían los filósofos modernos, claro que la posición tomada por estos puede ser entendida, a los ojos de este discurso, como un intento de contrarrestar la acción revolucionaria fingiendo una neutralidad que en realidad apoya la posición del soberano.

Si avanzamos un poco más en la genealogía elaborada por Foucault vemos como este discurso sufre, a partir del siglo XVIII dos transformaciones sustanciales. La primera de ellas sustituye la idea de raza por la de clase, y elabora así la idea de lucha de clases en el interior de las sociedades modernas - concepto típicamente marxista que en realidad debe mucho a los historiadores franceses, quienes fueron los primeros en elaborarlo -. Y la segunda transformación, la que nos resulta más interesante para el presente trabajo, se lleva a cabo cuando la idea de raza tomo un carácter de tipo biológico, en este momento nos encontramos efectivamente ante la visión de una raza 
superior, que es la que debe sobrevivir de acuerdo a los mandatos de la evolución, y una raza inferior condenada a la extinción. Además esta segunda transcripción del discurso de la lucha de razas aporta un nuevo elemento: mientras que en la teorización Ilevada a cabo en el siglo XVII ambas razas en conflicto se reconocían como ajenas una a la otra, como irreconciliables en algún elemento constitutivo de su cultura o su modo de ver el mundo, este nuevo discurso plantea una solo cuerpo social que se subdivide en su propio enemigo. "En otras palabras: lo que vemos como polaridad, como ruptura binaria en la sociedad, no es el enfrentamiento de dos razas recíprocamente exteriores; es el desdoblamiento de una única raza en una superraza y una subraza." (Foucault, 2000, p. 65)

\section{Racismo y biopolítica}

Hemos visto en el capítulo anterior como durante el siglo XVII - momento de su mayor desarrollo teórico mas no de su nacimiento - el discurso de la lucha de razas cobra relevancia como una herramienta para criticar el derecho soberano; hemos mencionado su función política y como hace de la sociedad un campo de batalla en el cual la historia y la verdad son armas fundamentales a disposición de todos los bandos implicados. También seguimos la evolución de este discurso hasta que el mismo adquiere, en el siglo XVIII y con más fuerza en el XIX, un carácter biologicista e impulsa a la sociedad a plegarse sobre sí mismo para encontrar allí, en su interior, el verdadero enemigo. Relacionemos ahora lo anterior con otro fenómeno que también tiene su génesis en el siglo XVIII: la biopolítica.

Para poder comprender mejor las implicaciones que planteó el surgimiento de la biopolítica creemos que es necesario, en primer término, retroceder algunos siglos. "Durante mucho tiempo, uno de los privilegios característicos del poder soberano fue el derecho de vida y muerte." (Foucault, 2014, p. 127) El filósofo francés relaciona este poder con la patria potestas romana, es decir, el derecho del padre de familia de disponer de la vida de sus hijos al igual que de la de sus esclavos. El soberano, el rey, gran padre figurativo de la sociedad, puede también disponer de la vida de sus súbditos si así lo considerase oportuno, si estuviese en riesgo su vida o sus 
propiedades. La ejecución pública es una forma de mostrar la fuerza de la venganza del soberano, y de modo similar, si la amenaza es externa, este puede disponer de la vida de sus súbditos de forma indirecta mandándolos a combatir en su nombre.

Ahora bien, a partir del siglo XVII - consolidándose en el XVIII - se da una transformación importante en esta forma de ejercer la soberanía:

"Esa muerte, que se fundaba en el derecho del soberano a defenderse, o a exigir ser defendido, apareció como el simple envés del derecho que posee el cuerpo social de asegurar su vida, mantenerla y desarrollarla." (Foucault, 2014, p. 129)

Los Estados a partir del siglo XVII pondrán en marcha diferentes estrategias con el objetivo de asegurar la supervivencia de la población. Esas estrategias se enmarcaron en dos polos que, como señala Foucault, no deben ser vistos como antitéticos, sino más bien como una continuación que se complementa y en la cual ninguno de los dos polos anula o excluye totalmente al otro.

El primer polo en surgir, en un sentido cronológico, es el que se enfoca en el cuerpo como máquina, en su sectorización, su disciplinamiento, su entrenamiento; dicho polo constituye la anatomopolítica del cuerpo humano. El segundo polo, el cual como hemos dicho - surge a mediados del siglo XVIII, se centra en el cuerpo-especie, es decir, ya no en los sujetos individuales, sino en en los fenómenos universales que atraviesan a todos los sujetos, es un polo centrado en el control de los nacimientos, las muertes, las enfermedades.

Centraremos nuestra atención en el fenómeno biopolítico, el cual hemos visto que surge en el mismo siglo que el discurso de lucha de razas de corte biológicoevolucionista. Las imbricaciones entre estos dos fenómenos no deben pasar inadvertidas, ni deben ser considerados como fenómenos paralelos, el racismo forma parte fundamental de la estructura estatal. "Desde el siglo XIX, los distintos regímenes políticos han puesto en marcha los formidables procedimientos de matanza incluso contra sus propias poblaciones." (Ruiz Gutiérrez, A., 2012, p. 61) Expresando su mayor fuerza en el siglo XX, época de los grandes genocidios dirigidos hacia sectores particulares de la población. Tomemos por ejemplo la eliminación programada de la 
población armenia, llevada a cabo por el imperio Turco-Otomano en 1915; el holocausto nazi, el cual tuvo sus mayores consecuencias durante 1941; o las matanzas perpetradas por los jemeres rojos en Camboya, las cuales iniciaron en 1975. Si sumásemos las víctimas solo de estos tres acontecimientos, la cifra asciende a un total aproximado de diez millones y medio de personas.

Creemos que estos dos fenómenos - el desarrollo de las medidas de tipo biopolítico y el racismo biológico evolucionista - son inseparables, pues de otro forma los fenómenos anteriores, y aun el que nos encontramos analizando, creemos que carecerían de sentido. En definitiva, una de las únicas formas de entender estas matanzas programadas por parte del poder soberano sobre su propia población es sobre la base del discurso de la lucha de razas del siglo XVIII: el enemigo no se encuentra en el exterior, el verdadero peligro se encuentra dentro de las fronteras de nuestro territorio: las razas inferiores. Y como un parásito que pone en riesgo la vida del cuerpo social, deben ser retirados del mismo. "El viejo poder soberano, que podía verter legítimamente la sangre de sus súbditos, se extiende al biopoder que hace morir a las razas inferiores a fin de dejar vivir a la superior" (Ruiz Gutiérrez, 2012, pp. 56 57)

Poco a poco contamos con más herramientas para interpretar el caso Rohingya desde una perspectiva biopolítica relacionada directamente con el discurso racista. Pero aun nos resta desarrollar dos conceptos que consideramos fundamentales: nuda vida y homo sacer.

\section{La biopolítica y el estado de excepción}

Si hablamos de Michel Foucault aun los más neófitos tienen una idea de quién fue este filósofo, y la clase de aportes que realizó. No ocurre lo mismo con Giorgio Agamben, motivo por el cual creemos necesario desarrollar un breve comentario sobre el mismo. En primera medida decir que es un filósofo nacido en 1942 y cuyos intereses teóricos se mueven por una extensa variedad de campos; el mismo aun está vivo y aun publica obras que se suman a un extenso catálogo. Entre estas encontramos las diferentes entregas de Homo Sacer, cuyo último libro en la serie se publico en el 2015, 
finalizando así un extenso recorrido teórico que nos brindará las herramientas faltantes para interpretar el éxodo Rohingya desde la perspectiva propuesta.

Nos centraremos en las primeras obras de la serie, en las cuales desarrolla los conceptos de nuda vida y homo sacer en relación al poder soberano. Pero en estos también dirige una pequeña crítica hacia Michel Foucault que creemos pertinente recoger. Siendo el filósofo francés el descubridor - al menos en tanto fue el primero en describir y estudiar el problema en profundidad - de la biopolítica, a Agamben le resulta interesante que el mismo nunca haya realizado un análisis profundo de los campos de concentración o de los grandes estados totalitarios del siglo XX, debido a que fue en estos estados de excepción donde más se puso en juego la vida de los sujetos. Utilicemos este comentario como excusa y desarrollemos una serie de conceptos fundamentales que se encuentran enunciados de forma apresurada en él, y de esta forma podremos entender en un sentido más completo la crítica y también avanzar en nuestro trabajo.

El primero de ellos nos obliga a remontarnos a la Antigua Grecia, el griego clásico poseía dos términos diferentes para referirse a la vida de los sujetos: por un lado bíos y por el otro zoé. El primero hace referencia a un determinado estilo de vida, cuando Sócrates en la Apología dice que una vida sin análisis no vale la pena ser vivida utiliza este término, y lo hace pues se está refiriendo al continuo proceso de vivir aplicando el análisis a uno y a los conciudadanos de la polis. El segundo término, en cambio, hace referencia a la vida biológica, privada de todo carácter político o social, la vida más natural, la vida privada de todo contenido, la vida al desnudo. "No obstante, en el mundo clásico, la simple vida natural es excluida del ámbito de la polis en sentido propio y queda confinada, en exclusiva, como mera vida reproductiva, en el ámbito de la oîkos." (Amgamben, 2010, p. 10). En contraposición a esto, el gran descubrimiento de Foucault es mostrar cómo, a partir de la modernidad, es esta vida natural la que entra en los planes y preocupaciones de los Estados. "Durante milenios, el hombre siguió siendo lo que era para Aristóteles: un animal viviente, y además capaz de una existencia política; el hombre moderno es un animal en cuya política está puesta en entredicho su vida de ser viviente." (Foucault, 2014, p. 135). 
Un segundo concepto fundamental en la obra de Agamben, y en nuestro desarrollo teórico, es el de estado de excepción. Siendo un tema muy complejo, trataremos de ser lo más precisos posibles dentro de los límites de la disponibilidad espacial del presente trabajo. El estado de excepción puede ser comprendido como el espacio - a veces geográfico, a veces temporal o ambos - en el cual las leyes se suspenden para solucionar algún problema interno. En Argentina poseemos la figura legal del "estado de sitio", el cual puede ser propuesto por el presidente y aprobado por el poder legislativo, para de esta forma suspender los derechos de los ciudadanos mientras se despliega a las fuerzas militares para contrarrestar las amenazas al territorio, esto fue lo ocurrido en diciembre del 2001, pero el estado de excepción es un concepto que va mucho más allá, tomemos este ejemplo solo como eso.

Carl Schmitt, filósofo jurídico alemán, definió al soberano como aquel que decide sobre el estado de excepción, y esto plantea una seria paradoja, la cual nos permite desarrollar una serie de postulados interesantes: el soberano está, al mismo tiempo, dentro y fuera del ordenamiento jurídico, puesto que es el único que puede suspender la validez de la ley debe encontrarse fuera de esta, pero a la vez, el soberano no puede estar completamente al exterior pues esta suspensión es una decisión legal, y este mantiene una estrecha relación con el ordenamiento jurídico. “El soberano, por medio del estado de excepción, 'crea y garantiza la situación' de la que el derecho tiene necesidad para su propia vigencia." (Agamben, 2010 , p. 29)

Hemos visto, citando a Foucault, que la inclusión de la vida natural en el ámbito político marca el paso de la época clásica a la modernidad. Agreguemos a eso y a lo último que hemos desarrollado que el estado de excepción no es solo una medida legal, sino que, dado que el soberano lo decreta para proteger a la sociedad (en el caso de una guerra, por ejemplo), es también una medida política. Así pues, se llega a la conclusión de que la vida se incluye en el ámbito político excluyéndose de este por el estado de excepción: esta es la situación que hemos mencionado en el párrafo anterior.

Esta última idea puede resultar compleja, así que detengámonos. Debemos caer en otra aparente paradoja para poder entender cómo es posible la inclusión de la 
vida en el estado de excepción: "[...] lo que caracteriza propiamente a la excepción es que lo excluido no queda por ello absolutamente privado de conexión con la norma" (Agamben, 2010, p. 30). El estado de excepción, entonces, no debe ser entendido como una situación caótica carente de normas, sino más bien como la de suspensión de estas, pero no por un potencia activa del estado de la excepción, sino por la retracción de las normas, la cual da lugar a aquel y permite que estas se constituyan como reglas.

Es decir, para que las normas sean aceptadas como reglas, estas deben mantenerse en relación directa con la excepción, y esta relación se da a través de la figura de la suspensión, entendida esta como el proceso por el cual las normas se retraen y dan lugar a la excepción. Podemos interpretar en esto un adentro y un afuera, en donde la suspensión permite que la norma se mantenga en relación con el afuera, y el estado de excepción el límite entre ambos. Ahora bien, las normas obtienen validez en esta suspensión debido a que la misma permite una relación no particular con un elemento externo al aparato legal, por ejemplo, la violencia entendida como estado de naturaleza, la suspensión permite que la norma se mantenga en contacto con esa figura y pueda aplicarse a los casos específicos que sean normalizados en el interior.

"La excepción es, en este sentido, la localización (Ortung) fundamental, que no se limita a distinguir lo que está dentro y lo que está fuera, la situación normal y el caos, sino que establece entre ellos un umbral (el estado de excepción) a partir del cual lo interior y lo exterior entran en esas complejas relaciones topológicas que hacen posible la validez del ordenamiento." (Agamben, 2010, p. 31)

Si hemos podido afirmar, siguiendo las palabras de Foucault, que lo que caracteriza a la modernidad, en términos políticos, es la introducción de la vida natural en las preocupaciones políticas, y siguiendo a Agamben hemos visto que el aparato legal y político sustentado por la excepción, se aplica desaplicándose, o en otros términos incluye excluyendo, nos resta determinar cómo se lleva a cabo esta inclusión de la vida en el aparato legal y político a partir de la exclusión de la misma. Y para tal 
fin, debemos recurrir a otro de los conceptos de Giorgio Agamben, en este caso el que da título a las obras que nos encontramos analizando: Homo sacer.

\section{El hombre sagrado}

"Mucho se ha debatido sobre el sentido de esta enigmática figura, en la que algunos han querido ver "la más antigua pena del derecho criminal romano'" (Agamben, 2010, p. 94). El homo sacer, como podemos determinar a partir de la cita anterior, es una oscura y compleja figura del derecho romano. Por un lado, es la primer vez en la historia en que el carácter de sacralidad se vincula a una vida humana, y por el otro, es un complejo concepto en el cual coinciden dos características que, a primera vista, parecen inconciliables.

El hombre sagrado, traducido literalmente, poseía una conexión directa con los dioses, pero pese a esto, el mismo podía ser asesinado por cualquier ciudadano sin que esto configurase un delito. A su vez, tampoco podía ser sacrificado en nombre de ninguna deidad. Es, por lo tanto, una figura que se sitúa fuera del derecho humano, en tanto cualquiera puede darle muerte sin recibir castigo, y fuera del derecho divino, pues no puede ser sacrificado. Nos encontramos con otra figura que se mueve en un limbo, con otra exclusión inclusiva.

Quizás lo primero que llama la atención respecto a esta figura es el hecho de que cualquiera puede darle muerte sin recibir por ello una pena o castigo, nos hace pensar en la pena capital y en qué relación tendrán estas dos figuras en el derecho romano. De hecho, la pena capital es un claro ejemplo de la doble situación de exclusión en la que se encuentra el homo sacer. Si un hombre incumplía sus obligaciones para con otros hombres o los dioses caía en una grave falta de la cual podía librarse a través de diferentes rituales arcaicos, en este sentido, la pena de muerte es entendida como un ritual de purificación, y como el homo sacer se encuentra fuera de la esfera divina no puede ser ejecutado.

Tenemos así una figura que es exterior tanto al derecho divino como al derecho humano, y en ese sentido plantea una situación similar a otra que hemos analizado previamente: la paradoja del soberano, el cual puede decidir sobre el estado de 
excepción, es decir, sobre la suspensión de las normas, desde el marco de un organismo legal. "Soberana es la esfera en que se puede matar sin cometer homicidio y sin celebrar un sacrificio; y sagrada, es decir, expuesta a que se le de muerte, pero insacrificable, es la vida que ha quedado prendida en esfera" (Agamben, 2010, p.109). Siguiendo estos desarrollos podemos afirmar que ante el soberano todos son homini sacri, y por el contrario, el homo sacer tiene a todos como soberanos.

En capítulos anteriores hemos mencionado a Thomás Hobbes y su conocido Leviathán como modelos de organización estatal, este modelo de organización se crea cuando los habitantes de una sociedad aceptan formar parte de un contrato colectivo por el cual cada uno cede parte de su libertad a un soberano, el cual será el encargado de proteger el cuerpo social, pues el hombre es por naturaleza codicioso y negativo para los otros hombres. Retomamos estos desarrollos de la filosofía clásica para analizarlos a la luz de lo que hemos venido desarrollando.

Los partidarios de las teorías contractualistas ven en este momento previo la fundación del posterior estado, pues bien, tenemos registros de una figura de la vida pre-social indoeuropea que se relaciona estrechamente con la figura del homo sacer: el hombre-lobo. Antes de convertirse en un monstruo arquetípico este ser formaba parte del derecho germánico primitivo, y al igual que el homo sacer, cualquiera podía darle muerte sin recibir un castigo a cambio y tampoco podía ser sacrificado. Era un excluido de la comunidad, un ser en el límite entre lo salvaje y lo humano, allí estriba su condición de hombre-lobo, de wargus: es, una vez más, una paradoja, una figura límbica que se mueve fuera y a la vez dentro de dos espacios.

Desde esta perspectiva podemos llegar a una comprensión quizás más profunda de la metáfora del estado de naturaleza hobbesiano, el mismo, como sabemos, se refiere a un pasado ideal - en tanto no real-histórico - en el cual los hombres competían entre si en una guerra de todos contra todos, situación que se resuelve por el contrato. Pero para nosotros el estado de naturaleza no será una condición prejurídica completamente indiferente al derecho de la ciudad, tendrá una íntima relación con el estado de excepción, será un momento de suspensión a partir del cual las normas adquieren su validez como leyes, y los hombres, más que en una 
situación de guerra de todos contra todos, se encuentran en un estado en el cual todos son wargus, homo sacer, para los demás. "Solo este umbral, que no es ni la simple vida natural ni la vida social, sino la nuda vida o la vida sagrada, es el presupuesto siempre presente y operante de la soberanía." (Agamben, 2010, pp. 137-138).

\section{Nuda vida}

Si ahora retomásemos la crítica de Agamben a Foucault seguramente la entenderíamos mejor, puesto que si el filósofo francés afirmaba que la modernidad se caracteriza por la entrada de la vida en la política, nada justifica - quizás solo la muerte temprana - que no incluyera en sus reflexiones los campos de concentración o los estados totalitarios, espacios en los cuales el poder soberano se ejerce directamente sobre la nuda vida, como hemos visto. Para finalizar el desarrollo teórico que nos hemos propuesto, nos resta explicar este concepto de nuda vida y relacionar todo lo dicho con el caso Rohingya.

La nuda vida es la vida natural que se incluye en el ordenamiento políticojurídico, y por lo tanto está fuera tanto de la esfera de lo natural como de lo social, no es bíos ni zoé, es un límite que incluye excluyendo a la una en la otra, y en este sentido puede ser definida también en los términos de un intersección.

La nuda vida se relaciona de forma directa, como hemos visto, con los conceptos de homo sacer u hombre-lobo, y se colocan en el fundamento del poder soberano: este último es el que puede decidir sobre el estado de excepción, en este estado todos los sujetos se convierten en nuda vida y, por lo tanto, el soberano puede disponer de sus vidas.

Los hombres, entendidos como nuda vida, se incluyen en las preocupaciones político-jurídicas a través de una exclusión, es la culminación, en cierto sentido, de la cadena de paradojas. Es en este sentido en el que la vida entra a partir del siglo XVIII de acuerdo a los desarrollos de Foucault, pero en realidad, como hemos visto, las figuras del homo sacer y del wargus datan de milenios en el pasado, así que más que una puesta en escena de la nuda vida a partir de la modernidad, deberíamos hablar de un des-cubrimiento de esta vida como fundamento de la soberanía. 
Recapitulando todo lo dicho en estos últimos párrafos, la inclusión de la zoé en el territorio jurídico-político se da a través del estado de excepción, entendiendo este como el umbral entre lo exterior y lo interior al aparato legal, asegurado por la suspensión de las leyes. La nuda vida, como concepto que incluye a las figuras del homo sacer y el wargus, aparece como la habitante de este espacio, y se encuentra, por lo tanto, incluida en el sistema político y legal a través de un exclusión del mismo. $\mathrm{Y}$ el soberano es la figura que puede tomar decisiones acerca de este estado de excepción y que, por este motivo, se encuentra también dentro y fuera del sistema.

Ahora bien, hemos dicho que el estado de excepción es una medida que el soberano debe tomar para poder proteger a la sociedad de algún peligro. Pero como hemos visto anteriormente, el discurso racista del siglo XVIII considera a determinados sectores de la sociedad - en un principio razas en sentido biológico, como en el ejemplo del genocidio armenio, y posteriormente sectores de la sociedad que presentan diferencias ideológicas, las cuales impiden la consecución de un determinado objetivo, como en el ejemplo de la matanza en Camboya perpetrada por los jemeres rojos - como enemigos.

"El totalitarismo moderno puede ser definido, en este sentido, como la instauración, a través del estado de excepción, de una guerra civil legal, que permite la eliminación física no solo de los adversarios políticos sino de categorías enteras de ciudadanos que por cualquier razón resultan no integrables en el sistema político." (Agamben, 2004, p. 25)

\section{El caso Rohingya desde una perspectiva biopolítica}

Visto a la luz de lo que hemos desarrollado en el presente trabajo, el caso Rohingya no debe parecernos una excepción al curso natural de las cosas, como uno podría pensar en un primer momento cuando lee las cifras del exilio y los asesinatos en tierras birmanas. Es un caso testigo de todo lo que hemos desarrollado, motivo por el cual es pertinente ahora realizar un resumen de lo expuesto, contrastándolo con las informaciones que hemos recogido del caso. 
Hemos visto que a partir de 1982 las autoridades de Birmania negaron el derecho de ciudadanía y con el todas las garantías previstas para los ciudadanos al colectivo Rohingya, a la vez que continuaban una cruenta campaña de asesinatos y persecuciones - esta vez con consecuencias cada vez mayores - excusándose en ataques pasados llevados a cabo por fuerzas insurgentes. Situación que se repitió, con resultados aun más sangrientos, desde octubre del 2016, lo que derivó en el éxodo masivo a mediados del año pasado. Si analizamos esto desde el discurso racista del siglo XVIII comprendemos claramente las verdaderas motivaciones del gobierno birmano: eliminar por completo un sector de la población que creían perjudicial para el bienestar del colectivo social, el cual apoyó mayoritariamente esta campaña de limpieza étnica. También bajo esta luz comprendemos los testimonios recogidos por Beech, H. (2017) en su artículo 'No such thing as Rohingya': Myanmar erases a history “'There is no such thing as Rohingya,' said U Kyaw San Hla, an officer in Rakhine's state security ministry. 'It is fake news.'”25 El concepto es eliminar, no solo al pueblo, sino toda su historia, pues esta, como vimos, tiene funciones políticas muy marcadas en el contexto de guerra de los discursos de verdad.

Los habitantes Rohingyas de Rakhine que han vivido asesinatos, incendios intencionales y campañas de violaciones, todos estos ejecutados por las fuerzas militares birmanas son ejemplos de nuda vida, habitando el estado de excepción que es su identidad desde 1982, momento en el cual se les negaron los derechos más indispensables, y fueron conservados en la sociedad a través de su exclusión de esta: ser Rohingya implicaba habitar en la excepción, ser wargus, homo sacer, nuda vida susceptible de ser eliminada por el poder soberano en cuanto este lo dispusiese.

Bajo estas dos figuras - estado de excepción y nuda vida - entendemos como es posible que todos estos abusos se hayan llevado a cabo sin ninguna pena a cambio, ni siquiera por parte de los organismos internacionales quienes han amenazado al gobierno de Birmania con duras sanciones, las cuales no han sido llevadas a término. Cualquiera podía tomar la vida de un Rohingya, incendiar su hogar y todo lo que

25 "'No existe tal cosa como Rohingya', dijo U Kyaw San Hla, un oficial del ministerio de seguridad del estado de Rakhine. 'Son noticias falsas'" 
poseían, o podían violar a sus mujeres sin que haya un castigo - es más, en algunas declaraciones, autoridades estatales y militares de Birmania han declarado que sería imposible que sus tropas realicen campañas de violaciones pues las mujeres Rohingya no son nada atractivas, lo que al parecer debería servir como una garantía de que tales hechos son noticias falsas - eran homini sacri.

A lo anterior agreguemos las justificaciones que se pueden desprender de la actitud de las autoridades birmanas. En primer lugar, recordemos, se cree que los Rohingya son un pueblo invasor que llegó a esas tierras durante el período de colonización británica. Pronto las acusaciones de ataques por parte de insurgentes de este sector minoritario llevaron a las fuerzas militares a actuar con brutalidad. Pero desde la perspectiva del discurso racista del siglo XVIII todo esto es, cuando menos, comprensible: el Estado birmano ve en los Rohingyas un enemigo, un impedimento al crecimiento que debe ser eliminado para de esta forma lograr la superviviencia del cuerpo social.

Y no debemos creer que este es un caso aislado, que cosas así solo ocurren en países remotos. Si nuestro desarrollo nos demuestra algo es que el racismo, la soberanía, la nuda vida y la biopolítica se relacionan estrechamente, y puesto que en la actualidad las preocupaciones estatales aun pasan por el control y cuidado de la vida, todos estos discursos se encuentran en la base de cada sociedad contemporánea.

Al leer las noticias acerca de algún atentado terrorista estamos viendo una lucha por parte de diferentes grupos, identificables como etnias antagónicas musulmanes contra occidentales normalmente - que se disputan el control de un territorio y el reconocimiento de sus derechos. La lucha, la sangre, son el fundamento de nuestras sociedades tal y como lo ha mostrado Foucault en sus análisis y Agamben al hablar de la suspensión de la ley como condición necesaria para que la norma sea reconocida como ley. Y una vez que las armas descansan la lucha continúa bajo la forma de producción de discursos: la otra cara de la brutalidad militar birmana son sus discursos tendientes a mostrar al pueblo Rohingya como invasores violentos, lo cual validaría su supresión. La producción de verdades entra en estrecha relación con la sangre derramada. 
La perspectiva biopolítica, atravesada por los diferentes análisis que hemos utilizado, es una herramienta indispensable para el diagnóstico de la situación actual de múltiples países y sociedades a lo largo y ancho del planeta. Fue Foucault quién inició la tarea de develar las estrategias del poder desarrollando, en el proceso, y de manera discontinua, sus posiciones sobre el racismo - decimos esto pues, aunque lo haya abordado en la obra que analizamos, también es un tema presente en sus libros sobre la sexualidad, la locura, la enfermedad o cualquiera en que una minoría es segregada por una mayoría - y Agamben el encargado de acercar estos desarrollos al ámbito de la soberanía y de los estados totalitarios, los cuales se disuelven para dar paso a las democracias, las cuales, en el fondo, funcionan de la misma forma: tomando a la nuda vida como fundamento del poder soberano.

\section{Conclusión}

Fue nuestro interés, a lo largo del presente artículo, mostrar que el análisis del caso Rohingya podía permitirnos mostrar que las regulaciones de un Estado pueden estar dirigidas a la desaparición de un pueblo entero. Habiendo analizado los diferentes aportes que Michel Foucault y Giorgio Agamben podían brindarnos en relación con este tema creemos que lo hemos cumplido.

El pueblo Rohingya, perseguido y quizás al borde de la desaparición, es un paradigma de la segregación racial contemporánea, y como tal, no debe pasar desapercibido. Quizás haya parecido que a la hora de enunciar los abusos por parte de las autoridades birmanas los naturalizábamos al ponerlos en relación con las teorías que se encuentran en el trasfondo, pero no fue esta nuestra intención, sino mostrar que estos, en cierto punto, no son diferentes de otros abusos llevados a cabo por otros Estados en otras partes del mundo, por ejemplo las limitaciones a la hora de acceder a una visa en Estados Unidos debido a sus nuevas políticas de inmigración, la discriminación social creciente hacia los habitantes musulmanes, o el arraigo cada vez mayor de los discursos políticos de ultraderecha en países como Bélgica o Austria.

Entendamos esto, lo que implica ser un paradigma de la segregación racial es mostrar que lo que ocurre en ese caso particular se aplica a todos los casos en los que 
hablamos de racismo contemporáneo, y viviendo en un mundo completamente globalizado y atravesado por las migraciones, el racismo es un tema cotidiano.

Quizás hubiera sido necesario una mayor extensión en el desarrollo de algunos temas, pero creemos que se ha logrado mostrar como todos los temas expuestos se imbrican mutuamente: El tema de fondo sobre el cual se apoyarán los demás es la biopolítica del siglo XVIII, el gran aporte de Foucault, que en pocas palabras se define como la entrada de la vida en los asuntos de la política. En un primer momento desarrollamos el discurso de la lucha de razas y hemos visto como este se bifurca en dos versiones, siendo la que a nosotros nos interesa la que surge también en el siglo XVIII: el racismo biológico-evolucionista; el que hayan nacido en el mismo año nos da la pauta de que hay una relación entre ambos, y esa sensación se confirma al pensar en los grandes genocidios del siglo XX, se extermina a una parte de la sociedad para que la otra sobreviva, hacer vivir y dejar morir. Luego debimos desarrollar las complejas nociones de estado de excepción, homo sacer y nuda vida, entre las cuales hay una fuerte relación que las hace indisociables, hemos visto como la nuda vida se pone en el fundamento de la soberanía y habita en el estado de excepción, y en este sentido, se relacionan directamente con el presupuesto general de la biopolítica: colocar a la vida en la escena política como actor principal.

Racismo y nuda vida son, pues, los elementos constitutivos de los intereses políticos contemporáneos, y esto es precisamente, lo que podemos ver al analizar las desventuras del pueblo Rohingya. Quizás, en este sentido, aun nos parezca un ejemplo extremo, pero este pueblo nos permite comprender la actualidad de los diferentes conceptos y aportes teóricos realizados. No basta con pensar que los estados de excepción finalizaron con la caída de los grandes estados totalitarios, debemos actualizar estos conceptos, o al menos, ponerlos en relación con fenómenos contemporáneos.

Creemos que de este modo hemos dado un gran aporte a la hora de analizar el funcionamiento de los estados contemporáneos, des-cubriendo el rol que ocupa el racismo en estos, y como este se encuentra en íntima relación con la biopolítica. Es más, podríamos afirmar que mientras la biopolítica sea el paradigma del poder 
soberano, el racismo estará entre sus herramientas, justificando los abusos y las violencias de aquel sobre las nudas vidas abandonadas a su merced. Quizás el presente trabajo no tenga el peso de los autores que cita, pero estamos seguros de que brinda recursos para futuros análisis más profundos acerca de todas estas problemáticas y la mutua interrelación que hemos mostrado entre ellos.

\section{Bibliografía}

- AA. VV. (11 de enero del 2018). 'Beyond comprehension': Myanmar admits killing Rohingya. Al Jazeera. Recuperado de http://www.aljazeera.com/news/2018/01/rohingya-crisis-myanmar-militaryadmits-killings-180111102253768.html

- AA. VV. (28 de septiembre del 2017). Myanmar: Who are the Rohingya? Al Jazeera. Recuperado de http://www.aljazeera.com/indepth/features/2017/08/rohingya-muslims170831065142812.html

- AA. VV. (6 de octubre del 2017). Bangladesh to build one of world's largest refugee camps for 800,000 Rohingya. The Guardian. Recuperado de https://www.theguardian.com/world/2017/oct/06/bangladesh-build-worldslargest-refugee-camps-800000-rohingya

- Agamben, G. (2004). Estado de excepción. Homo sacer, II, I. Buenos Aires, Argentina: Adriana Hidalgo editora

- Agamben, G. (2010). Homo sacer. El poder soberano y la nuda vida I. Valencia, España: Pre-textos

- Beech, H. (2 de diciembre del 2017). 'No Such Thing as Rohingya': Myanmar Erases a History. The New York Times. Recuperado de https://www.nytimes.com/2017/12/02/world/asia/myanmar-rohingya-denialhistory.html

- Foucault, M. (1992). Microfísica del poder. Madrid, España: La Piqueta 
- Foucault, M. (2000). Defender la sociedad. Curso en el Collège de France (1975 - 1976). Buenos Aires, Argentina: Fondo de Cultura Económica de Argentina

- Foucault, M. (2014). Historia de la sexualidad. 1. La voluntad de saber. Buenos Aires, Argentina: Siglo veintiuno editores

- Gómez Izquierdo, J. (2014). La conceptualización del racismo en Michel Foucault. Interdisciplina, 2 (número 4). pp. 121-142 Recuperado de revistas.unam.mx/index.php/inter/article/download/47765/42949

- Judah, J. (11 de septiembre del 2017). Soaked and hungry Rohingya refugees seek shelter in Bangladesh. UNHCR The UN Refugee Agency. Recuperado de http://www.unhcr.org/en-us/news/latest/2017/9/59b6c0984/soaked-hungryrohingya-refugees-seek-shelter-bangladesh.html

- Landler, M. (22 de noviembre del 2017). Myanmar's Crackdown on Rohingya Is Ethnic Cleansing, Tillerson Says. The New York Times. Recuperado de https://www.nytimes.com/2017/11/22/us/politics/tillerson-myanmarrohingya-ethnic-cleansing.html

- Nurul Islam (5 de octubre del 2006). Facts about the Rohingya muslims of Arakan. Arakan Rohingya National Organisation. Recuperado de http://www.rohingya.org/portal/index.php/learn-about-rohingya.html

- Ruiz Gutiérrez, A. (2012). La nuda vida: entre el poder soberano y una analítica del biopoder. Logos. 2 (número 22). pp. 55 - 73 